\title{
Effects of disruptive selection XI: gene flow and divergence
}

\author{
C. G. N. Mascie-Taylor*, \\ J. B. Gibsont and J. M. Thoday
}

Department of Genetics, Downing Street, Cambridge CB2 3EH

Disruptive selection lines using sternopleural chaeta number in Drosophila melanogaster were run using weaker selection than hitherto. In addition, the design allowed some assessment of the degree to which partial isolation arising from distance, or other factors, increases the effects of disruptive selection. In all lines, even those with selection only eliminating 20 per cent of the flies in each generation, variance and the mean difference between extreme cultures increased, and in all lines isolation between the high and low subpopulations reached the maximum allowed by the experimental design. Reducing migration between the subpopulations from 50 per cent to 30 per cent of the parents in each generation had little effect, but further reduction made disruptive selection more effective. At the same time quite low migration reduced the effects of selection markedly.

\section{INTRODUCTION}

The experiments described in this paper are similar in basic design to those reported by Gibson and Thoday (1963, 1964). They explore the results of exposing populations to a model situation analogous to two adjacent environments which are within migration range of one another, and in which selection promotes opposite phenotypes. The two environments may be regarded as adjacent components of an environmental cline or as adjacent components of a mosaic of different niches.

In such a situation we wish to know:

(a) Whether the differential selection can be effective in establishing a phenotypic difference between the two subpopulations in the two environments, or whether gene migration prevents this.

(b) The relationships between the amount of migration, the strength of selection and the degree of divergence of phenotype.

(c) The effect of selection, and of response to selection, on the amount of migration, (that is, on the degree of isolation) between the subpopulations.

Present addresses: * Department of Physical Anthropology, Downing Street, Cambridge.

$\dagger$ Department of Population Biology, Research School of Biological Sciences, Box 4 P.O., Canberra, A.C.T., Australia.
This latter point needs illustration, which is done in fig. 1.

In this figure it is assumed that two subpopulations have different means, but mate at random, so that the total population contains 50 per cent hybrids between these two. It is easy to see that strong disruptive selection will eliminate many hybrids and most non-hybrid migrants, whereas weak selection will permit much gene flow between the two subpopulations of selected flies.

Gibson and Thoday's two experiments fitted this model closely. The strength of selection in the first was that implied by eliminating the intermediate 80 per cent of flies in each generation, and the second 50 per cent. In each of these experiments total variance rapidly increased, the subpopulations rapidly diverged in mean, and they rapidly became isolated from one another because the "hybrid" flies ceased to be included amongst those selected. (These experiments are not to be confused with those reported by Thoday and Gibson (1962) in which mating choice was allowed and strong reproductive isolation developed).

Though Gibson and Thoday's second experiment involved relatively weak selection, it seemed desirable to test the effectiveness of still weaker selection, and that was the prime purpose of the present experiments. However, in a realistic situation, it is to be supposed that microgeographic 


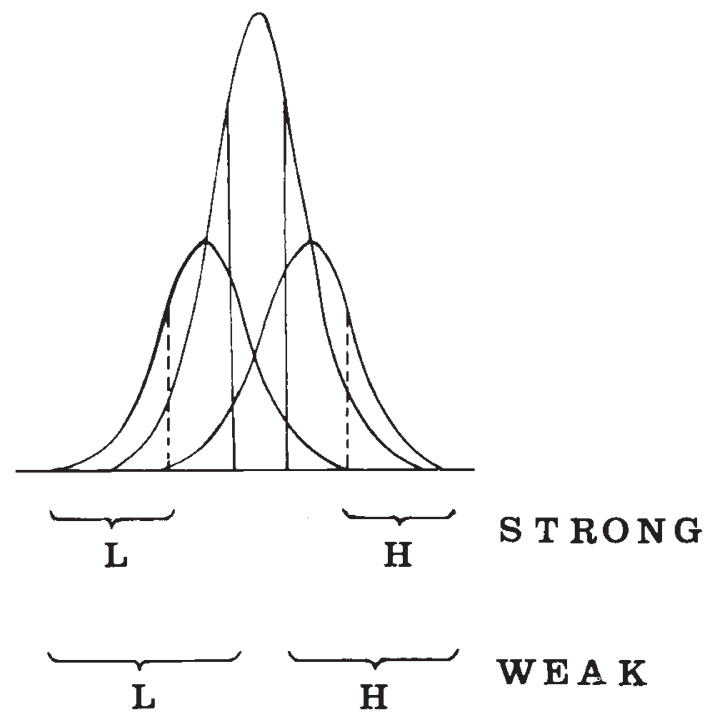

Figure 1 This presents three normal distributions. The central distribution contains twice the number of individuals as either of the others, as in our experiments in which 50 per cent of the individuals assayed before selection are hybrids. At the left is the distribution for progeny of $L \times L$ matings, at the right of $H \times H$ matings, and at the centre of $H \times L$ and $L \times H$ matings combined. The individuals rejected by weak disruptive selection are in the area between the solid vertical lines. Stronger selection might involve rejection of those between the broken lines. The figure illustrates how stronger selection implies greater isolation between the $H \times H$ and $L \times L$ sub-populations. It is also evident that increase of the difference distinguishing the $H \times H$ and $L \times L$ progenies, as a result of selection, must also increase isolation. It is also evident that reducing the number of hybrids assayed relative to $H \times H$ and $L \times L$ progeny will also increase such isolation.

distance separating the component environments would impose some measure of assortative mating (equivalent to reduced migration or initial partial isolation), and the experiments were designed to test the effects of this also. This is acheived by reducing the proportion of hybrids in the sample from which the selected parents are chosen. All the experiments involve symmetrical selection (see Thoday, 1972) that is to say that the $H \times H$ and $L \times L$ cultures contribute equal numbers to the sample, as if the two environments had equal carrying capacity.

\section{MATERIAL}

The selection lines were established from a wild stock of Drosophila melanogaster captured at Little Eversden near Cambridge in 1970. The variable was sternopleural chaeta number. The Eversden stock had a variance of $2 \cdot 77$, and heritability of $0.49 \pm 0.05$ as assessed by parent-offspring regression with assortative mating. The selection experiments began in June 1972 and continued to August 1973.

\section{DESIGN OF SELECTION LINES}

The mating and selection system was based on the quasi-random mating system of Thoday and Boam (1961) as used by Gibson and Thoday $(1963,1964)$.

To maintain a line in such experiments involves establishing four cultures, assaying a standard number of each sex from each culture, collecting virgins, selecting the required proportion of high and of low flies from the four progenies combined, and then setting up four new cultures with the four different matings $H q \times H \delta, H q \times L \delta, L q \times H \delta$, $L q \times L \delta$ and repeating the process each generation. In Gibson and Thoday's and the experiments reported here, each culture has four pairs of parents, so that $16 \mathrm{High}$ and 16 Low flies are selected as parents for each generation. For example, of the $8 \mathrm{H}$ females, allocation of four to the $H \times H$ cultures and four to the $H \times L$ cultures is at random. We shall, for convenience refer to the $H \times L$ and $L \times H$ cultures as "hybrid".

The variables in these experiments are the proportion of flies selected and the number of flies from the $H \times H$ and $L \times L$ cultures relative to those from the $H \times L$ and $L \times H$ cultures included in the sample from which selection is made. The proportion of flies selected is determined by the number of flies assayed before the 32 selected flies are chosen, and this controls the strength of selection. The relative number of $H \times H+L \times L$ to $H \times L+$ $L \times H$ culture flies assayed before selection affects the opportunity for migration in the experiment; reducing it below 50 per cent "hybrids" is analagous to introducing, from the beginning of the experiment, an element of assortative mating arising either from microgeographic or ecological partial isolation, or from some form of mating preference.

In Gibson and Thoday's experiments, equal numbers of flies from each culture were assayed before selection, and in the first 20 per cent and the second 50 per cent of the flies assayed were selected. We have run two replicate experiments with 50 per cent selected as the basis of comparison with their results, and with our other lines. In these two lines 50 per cent of the flies assayed came from the $H \times L+L \times H$ cultures. In the remaining lines less than 50 per cent of the flies assayed were 
rejected, and in some less than 50 per cent came from the "hybrid" cultures.

Table 1 details the design of the different lines. It should be noted that the number of flies in the sample assayed before selection given in this table is, of course, not the actual number assayed to assess the mean chaeta number of each culture. This was always 12 of each sex from each culture. Among this 12 the flies to be includec in the pre-selection sample were chosen by random numbers.

Table 1 The number of flies of each sex from the progeny of each culture that are included in the sample from which the 8 highest and 8 lowest are chosen to continue the line

\begin{tabular}{lrlll}
\hline & \multicolumn{4}{c}{ Culture } \\
\cline { 2 - 5 } Line designation* & $H \times H$ & $H \times L$ & $L \times H$ & $L \times L$ \\
\hline 50S/50H A and B $¥$ & 8 & 8 & 8 & 8 \\
$67 \mathrm{~S} / 50 \mathrm{H} \mathrm{A}$ and B & 6 & 6 & 6 & $6 \dagger$ \\
$67 \mathrm{~S} / 33 \mathrm{H} \mathrm{A}$ and B & 8 & 4 & 4 & 8 \\
$67 \mathrm{~S} / 17 \mathrm{H} \mathrm{A}$ and B & 10 & 2 & 2 & 10 \\
$67 \mathrm{~S} / 8 \mathrm{H} \mathrm{A}$ and B & 11 & 1 & 1 & 11 \\
$80 \mathrm{~S} / 50 \mathrm{H}$ A and B & 5 & 5 & 5 & $5 \dagger$ \\
$80 \mathrm{~S} / 30 \mathrm{H} \mathrm{A}$ and B & 7 & 3 & 3 & $7 \dagger$ \\
$80 \mathrm{~S} / 10 \mathrm{H} \mathrm{A}$ and B & 9 & 1 & 1 & 9
\end{tabular}

* The line designation shows the percentage of assayed flies chosen to continue the line, and the percentage of assayed flies that come from the "hybrid" cultures.

$\dagger$ It is important to note in these lines the selected sample must include flies from the hybrid cultures. In the remaining lines the selected flies could all come from $H \times H$ and $L \times i$ cultures. $\ddagger A$ and $B$ are replicate lines. Only the results of the A replicate are given in figs. $2-3$.

\section{RESULTS}

\section{Selection responses}

We present the results for each line in three forms. The first gives the means of the four cultures for each line in each generation. The second gives the proportion of flies from the hybrid cultures, that were included in the sample after selection. The third gives the histograms of chaeta number for all of the lines at the end of the selection programme. A and B are replicate lines. In view of the number of figures, and the fact that replicate experiments were essentially alike, we only give the detailed resuits of the A replicate of each type of line. Those who wish to see the detailed differen. ces between replicates are referred to MascieTaylor (1977). The figures which show the degree of isolation do however include both replicate lines.

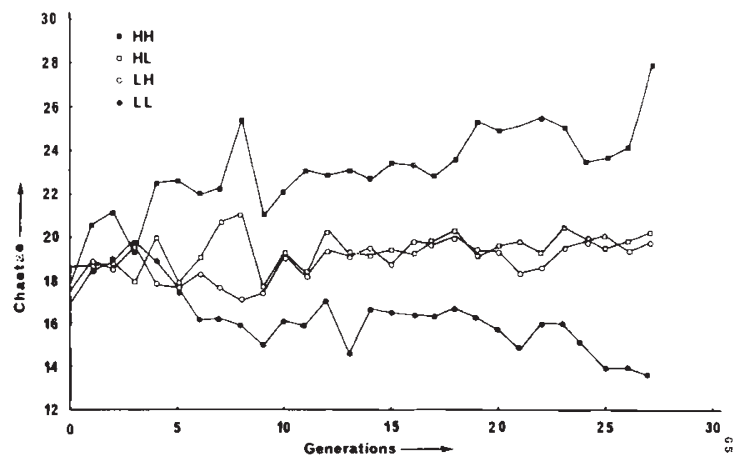

(a)

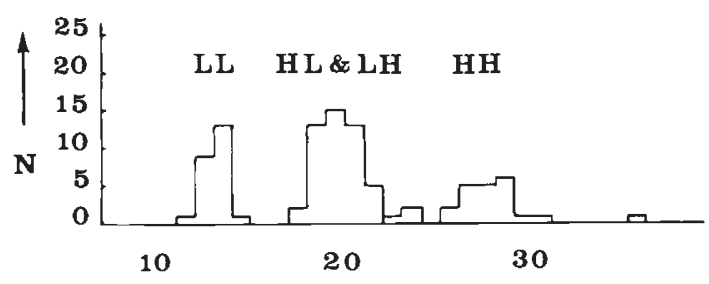

CHAETA NUMBER

(b)

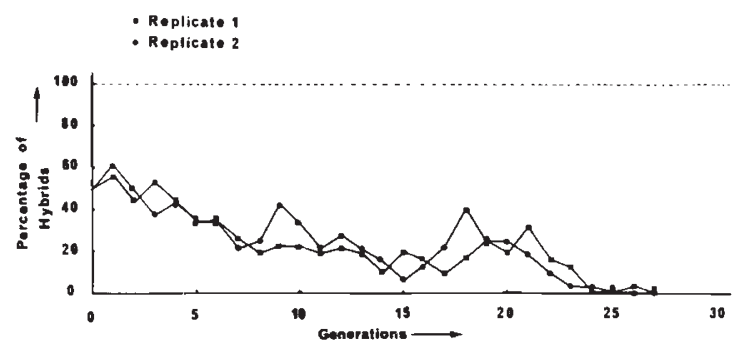

(c)

Figure 2 The 50S, 50H lines. (a), Means; (b), Histogram at the end of the experiment; (c) The percentage of the selected flies that come from the hybrid cultures in each generation of the two replicate lines. The dotted line shows the maximum number of hybrid flies that could be selected.

The results may be summed up as follows:

(a) In all experiments the lines responded to selection in as much as overall variance increased and a difference of mean between the $H \times H$ and $L \times L$ cultures developed and in many steadily increased.

(b) In each experiment this difference is such that at the end there is very little overlap between the distributions of chatae number of the hybrid and ron-hybrid cultures.

(c) In each experiment the number of hybrids included in the selected sample has been reduced 


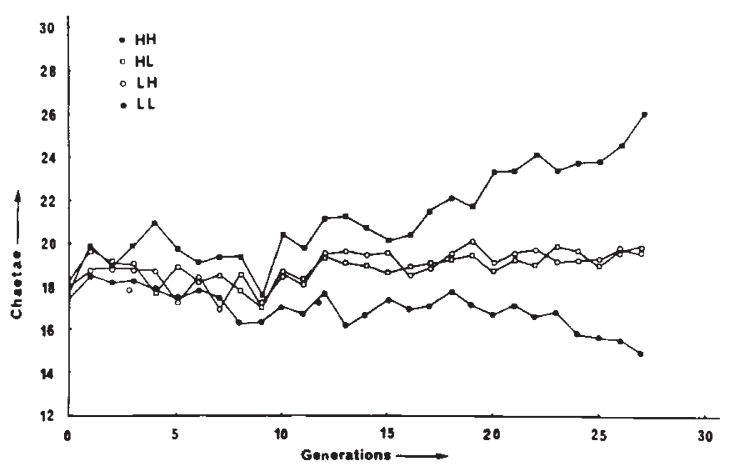

(a)

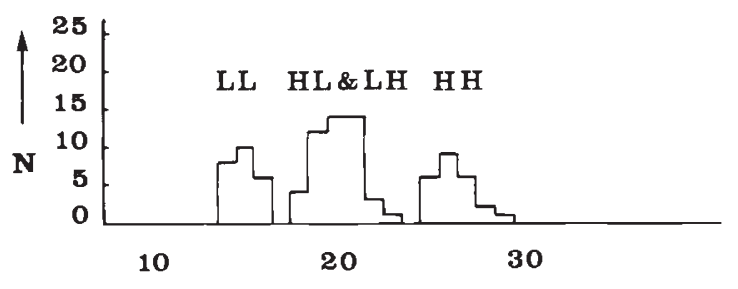

CHAETA NUMBER

(b)

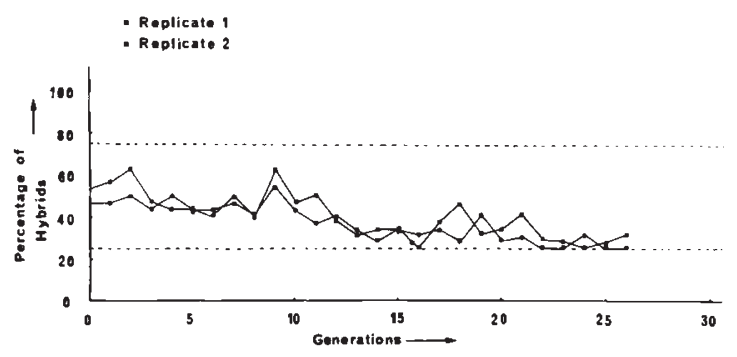

(c)

Figure 3 The 67S, $50 \mathrm{H}$ lines. (a), Means; (b), Histogram at the end of the experiment; (c), The percentage of the selected flies that come from the hybrid cultures. The upper dotted line shows the maximum number of hybrid flies that could be selected. The lower line shows the minimum number of hybrid flies that must be selected.

and in most it approached or reached the minimum possible allowed by the experimental design. Where this minimum is zero, the $H \times H$ and $L \times L$ cultures became isolated from one another.

(d) As might have been expected the difference of mean distinguishing the $H \times H$ from the $L \times L$ cultures increases with the strength of selection and decreases with the proportion of hybrids included among the flies from which selection is made.

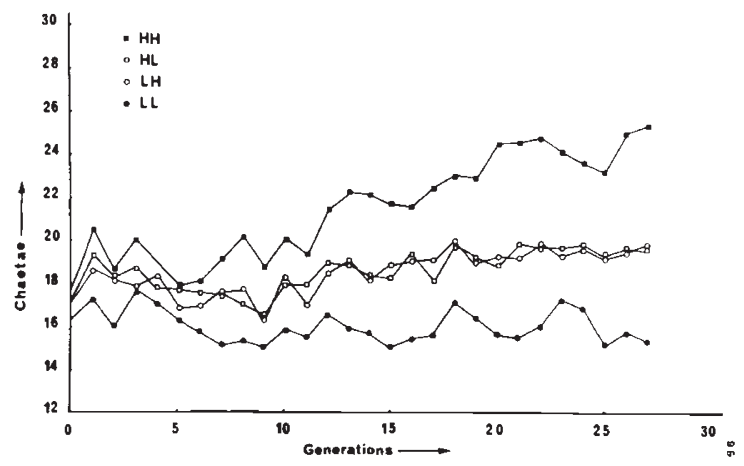

(a)

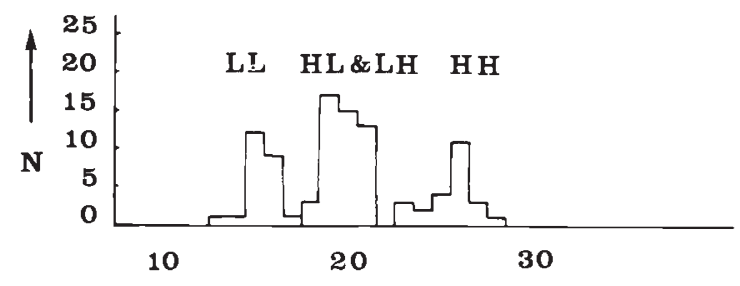

CHAETA NUMBER

(b)

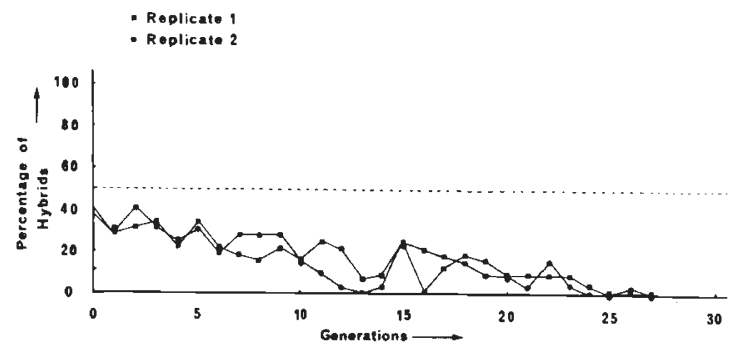

(c)

Figure 4 The 67S, 33H lines. (a), Means; (b), Histogram at the end of the experiment; (c), The percentage of the selected flies that come from the hybrid cultures in each generation of the two replicate lines. The dotted line shows the maximum number of hybrid flies that could be selected.

Fig. 10 illustrates point (d). The data are of course very limited because of the limited number of replicate lines it was possible to maintain, but it seems that the effects of the relative isolation imposed by reducing the number of hybrid flies available for selection, is negligible until they form less than 30 per cent of the sample, and is quite strong below this.

\section{The effect of gene flow}

In order to test whether gene exchange between the $H \times H$ and $L \times L$ cultures was significantly 


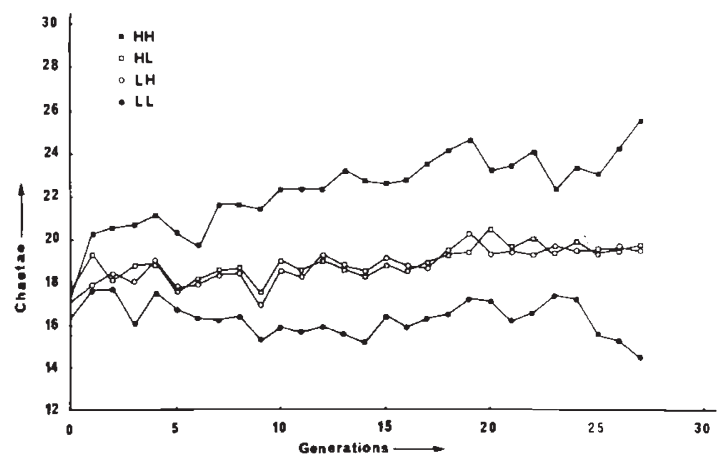

(a)

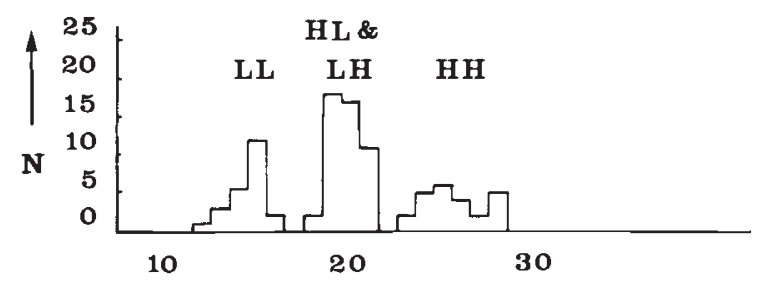

CHAETA NUMBER

(b)

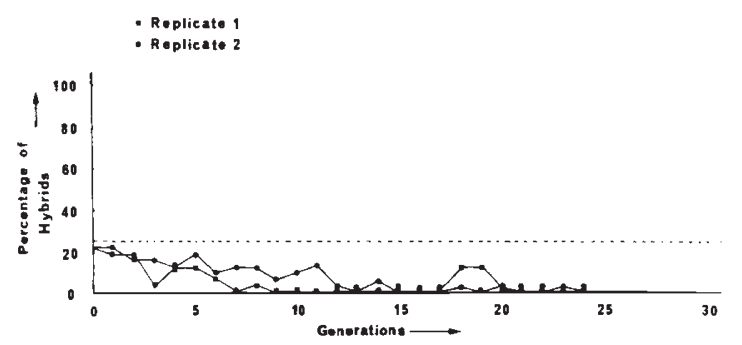

(c)

Figure 5 The 67S, 17H lines. (a), Means; (b), Histogram at the end of the experiment; (c), The percentage of the selected flies that come from the hybrid cultures in each generation of the two replicate lines. The dotted line shows the maximum number of hybrid flies that could be selected.

limiting the response to selection as assessed by their divergence in mean, additional sub-lines were taken from the $80 \mathrm{~S}$ lines and put under simple divergent directional selection, by reducing the number of flies from the hybrid cultures to zero. The results are included in Figs 2-9 and summarised in Fig. 10. It is clear that the forced hybridisation in these lines limited their response to selection, substantially, as was to be expected. The indications are however, that, even with the weak selection implied by elimination of only 20

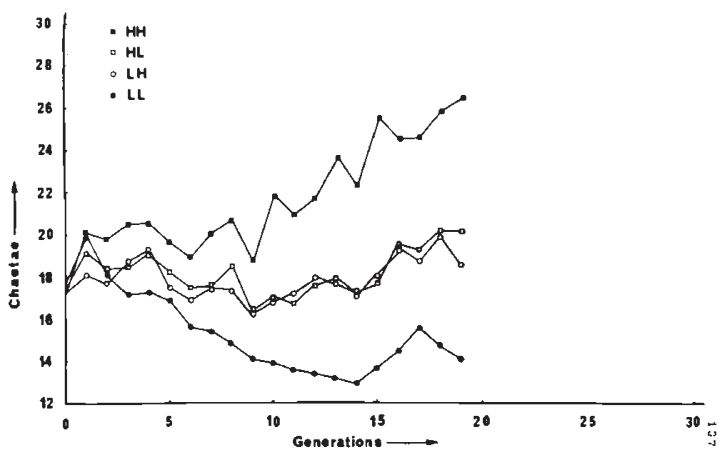

(a)

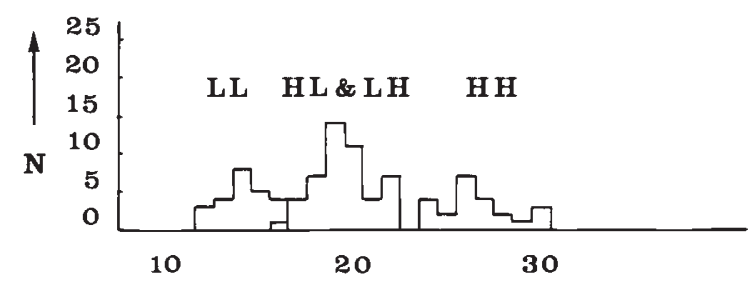

CHAETA NUMBER

(b)

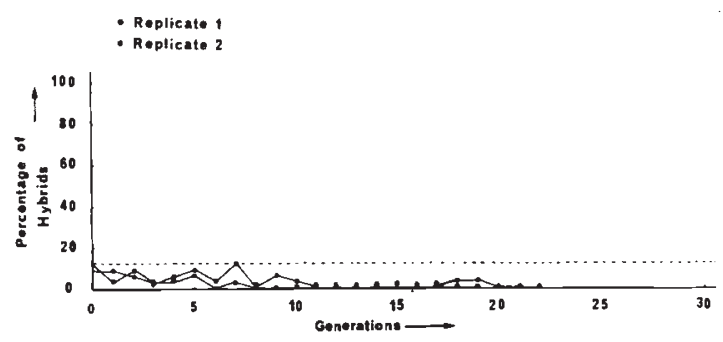

(c)

Figure 6 The 67S, $8 \mathrm{H}$ lines. (a), Means; (b), Histogram at the end of the experiment; (c), The percentage of the selected flies that come from the hybrid cultures in each generation of the two replicate lines. The dotted line shows the maximum number of hybrid flies that could be selected.

per cent of each generation, disruptive selection induces divergence more effectively than gene flow, as a counter force, induces convergence of the two subpopulations, and that if gene flow is reduced by including less than 30 per cent hybrids in the sample assayed before selection, even such weak selection can be very effective. In fact 7 generations of selection without migration had much less effect on the $80 \mathrm{~S} 10 \mathrm{H}$ lines than the others, showing how effective the $10 \mathrm{H}$ conditions allowed the low (80S) selection to be. 


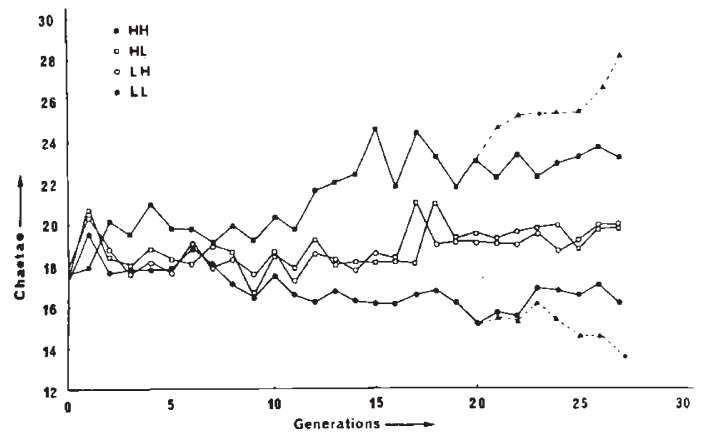

(a)

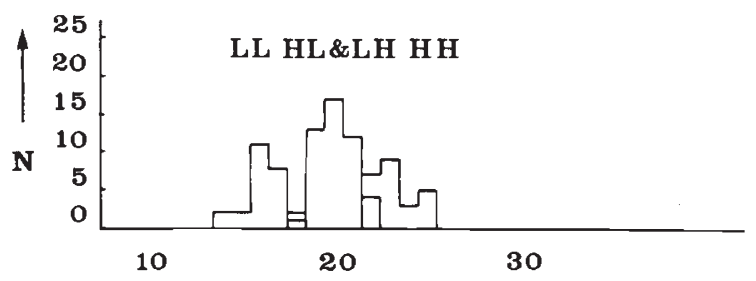

CHAETA NUMBER

(b)

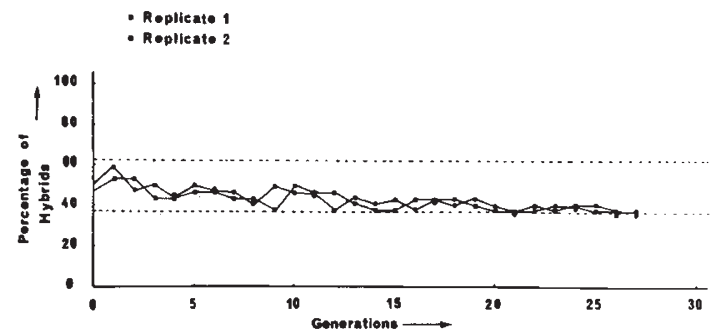

(c)

Figure 7 The $80 \mathrm{~S}, 50 \mathrm{H}$ lines. In (a) and (b) the dotted lines show the divergence when migration was reduced to zero (see text); (c), The percentage of the selected flies that come from the hybrid cultures in each generation of the two replicate lines. The upper dotted line shows the maximum number of hybrid flies that could be selected. The lower line shows the minimum number of hybrid flies that must be selected.

\section{DISCUSSION}

In Mather's original papers on Disruptive Selection (1955) he discussed its probable cutcome in two extreme conditions. In the first the two (or more) subpopulations selected were envisaged as dependent on one another, or tied together, through the breeding system, as are sexes, incompatability types, etc. The expected outcome was polymorphism. In the second they were not interdependent and the expected outcome was isolation.

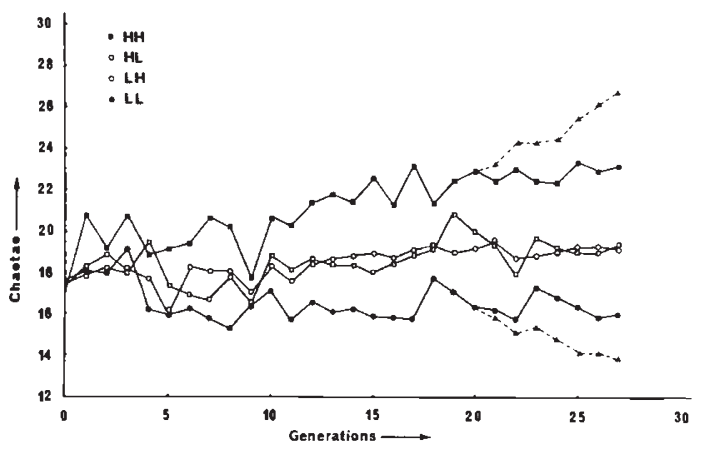

(a)

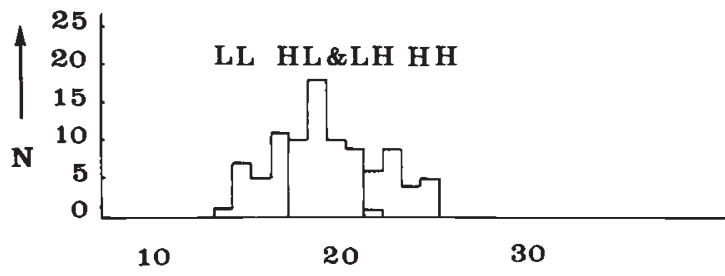

CHAETA NUMBER

(b)

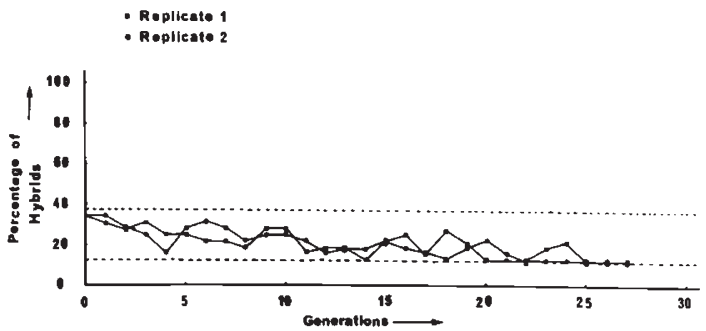

(c)

Figure 8 The $80 \mathrm{~S}, 30 \mathrm{H}$ lines. In (a) and (b) the dotted lines show the divergence when migration was reduced to zero (see text); (c), The percentage of the selected flies that come from the hybrid cultures in each generation of the two replicate lines. The upper dotted line shows the maximum number of hybrid flies that could be selected. The lower line shows the minimum number of hybrid flies that must be selected.

Ample evidence has been provided that both these conditions are possible, (see Thoday, 1972, for review). But the question when isolation or polymorphism are the more probable outcomes of disruptive selection when the two selected subpopulations are not interdependent is complex (see Thoday and Gibson, 1970).

The present experiments show that very weak disruptive selection can have powerful effects, and that, if microgeographic isolation were such that less than 30 per cent of any generation are hybrids between two slibpopulations, weak disruptive 


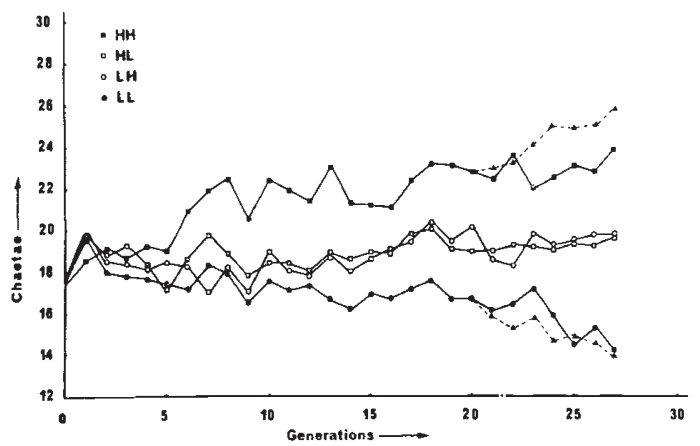

(a)

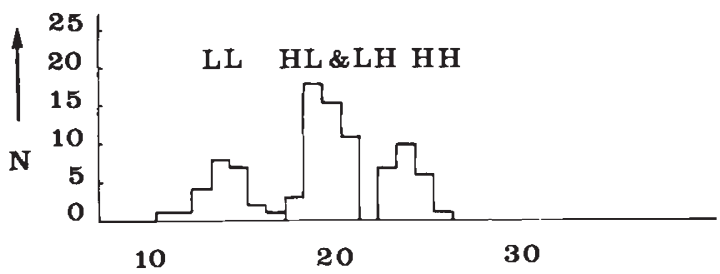

CHAETA NUMBER

(b)

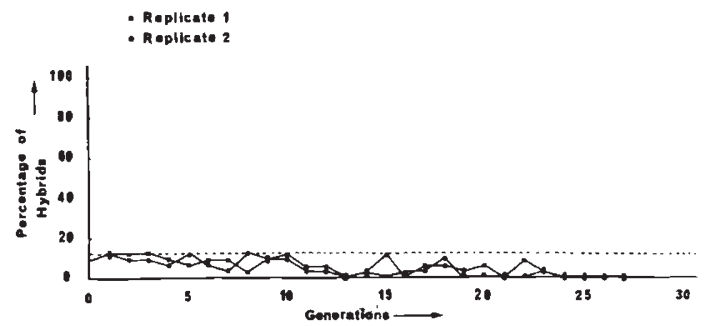

(c)

Figure 9 The $80 \mathrm{~S}, 10 \mathrm{H}$ lines. In (a) and (b) the dotted lines show the divergence when migration was reduced to zero (see text); (c), The percentage of the selected flies that come from the hybrid cultures in each generation of the two replicate lines. The dotted line shows the maximum number of hybrid flies that could be selected.

selection can be very effective, and can actually lead to the elimination of all hybrids from each generation, hence reinforcing the microgeographic isolation and making it complete.

This demonstration of the effectiveness of quite weak selection coupled with partial isolation resulting from microgeographic separation, reinforces the view that disruptive selection must play a part in the maintenance of genetic variation, the establishment of genetic variation, the establishment of polymorphisms, and the development of isolation in natural populations.

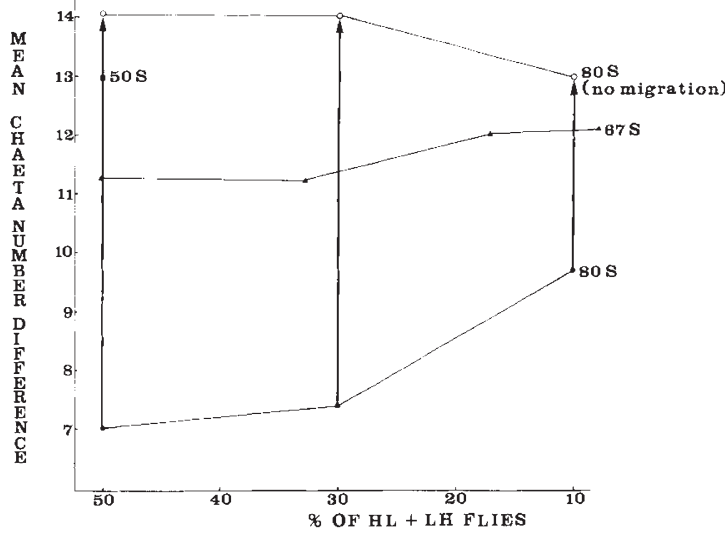

Figure 10 Differences in mean chaeta number between the progeny of $H \times H$ and $L \times L$ cultures averaged over the various pairs of lines at the end of the experiments. The solid square represents the lines in which 50 per cent of the flies were selected, solid triangles the four pairs of lines in which 67 per cent of the flies were selected and solid circles the 3 pairs of lines in which 80 per cent of the flies were selected. It can be seen that reducing the sample of hybrids from 50 to $30 \%$ has little effect, but less migration enables greater divergence of the $H \times H$ from the $L \times L$ culture means. Open circles show the divergences of the lines in which 80 per cent of the flies were selected after seven generations without migration. The arrows connect these points to the closed circles and show how effective the migration was in limiting divergence in those lines (see Tabie 1) in which hybrids were necessarily included among the parents at each generation.

\section{REFERENCES}

GIBSON, J. B. AND THODAY, J. M. 1963, Effects of disruptive selection. VIII. Imposed quasi-random mating. Heredity, $18,513-524$.

GIBSON, J. B. AND THODAY, J. M. 1964. Effects of disruptive selection. IX. Low selection intensity. Heredity, 19, 125130.

MASCIE-TAYLOR, C. G. N. 1977. Gene Flow and Divergence in Drosophila and Man. Unpublished Ph.D. thesis.

MATHER, K. 1955. Polymorphism as an outcome of Disruptive Selection. Evolution, 9, 52-61.

THODAY, J. M. AND GIBSON, J. B. 1962. Isolation by disruptive selection. Nature, Lond., 193, 1164-1166.

THODAY, J. M. 1972. Review Lecture: Disruptive selection. Proc. R. Soc. Lond. B, 182, 109-143.

THODAY, J. M. AND BOAM, T. B. 1961. Effects of disruptive selection. V. Quasi-random mating Heredity, 16, 219-223.

THODAY, J. M. AND GIBSON, J. B. 1970. The probability of isolation by disruptive selection. Am. Nat., 104, 219-230. 